\title{
Papanicolau e a saúde da mulher: importância do fomento à prevenção do câncer de colo uterino
}

Pap smear and women's health: importance of promoting cervical cancer prevention

Papanicolaou y salud de la mujer: importancia de promover la prevención del cáncer de cuello uterino

Amanda Guimarães Cunha ORCID: https://orcid.org/0000-0002-0893-3281

Centro Universitário Fibra, Brasil

E-mail: amandaguic18@gmail.com

Amanda Gomes Diniz Pimenta

ORCID: https://orcid.org/0000-0001-7098-7224 Universidade do Estado do Pará, Brasil

E-mail: amandagdpepper@gmail.com

Anne Letice Soares Braga

ORCID: https://orcid.org/0000-0002-1784-7414 Universidade Federal do Pará, Brasil

E-mail: annebraga0106@gmail.com

Alecsandra Jayná da Silva Cardoso

ORCID: https://orcid.org/0000-0001-9033-0641 Universidade Federal do Pará, Brasil

E-mail: alecsandrajayna@gmail.com

Bárbara Lima Santos

ORCID: https://orcid.org/0000-0001-6442-1806 Universidade Federal do Pará, Brasil

E-mail: barbaralima.enf@gmail.com

Camila de Paula Sousa da Rocha

ORCID: https://orcid.org/0000-0003-0129-228X

Universidade da Amazônia, Brasil

E-mail: kmilla_rochasfx@ hotmail.com

Daylane Pereira de Carvalho

ORCID: https://orcid.org/0000-0002-0489-4887

Centro Universitário Fibra, Brasil

E-mail: daylanecarvalhoatm@gmail.com

Denize Cardoso Portilho

ORCID: https://orcid.org/0000-0003-1754-1498 Universidade do Estado do Pará, Brasil E-mail: denizeportilho60@gmail.com

Daheny Coelho Matos

ORCID: https://orcid.org/0000-0002-8152-0256

Universidade do Estado do Pará,Brasil

E-mail: dahen.coelhomatos@gmail.com

Elisa da Silva Menezes

ORCID: https://orcid.org/0000-0002-8686-0727

Universidade da Amazônia, Brasil

E-mail: elisamenezes19@gmail.com

Jéssica dos Santos Vieira

ORCID: https://orcid.org/0000-0001-5083-6429

Escola Superior da Amazônia, Brasil

E-mail: jessicasv.enf.2020@gmail.com

Jéssica Maria Lins da Silva

ORCID: https://orcid.org/0000-0003-3218-6447

Universidade do Estado do Pará, Brasil E-mail: jeeh.sylva@gmail.com

Lucinéia Ferreira Ferreira

ORCID: https://orcid.org/0000-0001-8957-1536

Universidade do Estado do Pará, Brasil

E-mail: lucineiaferreira4509@gmail.com

Mônica Monteiro Ribeiro

ORCID: https://orcid.org/0000-0002-3823-5302

Faculdade Cosmopolita, Brasil

E-mail: monicamonrib@gmail.com 


\title{
Resumo
}

O câncer cervical é uma neoplasia maligna que ocupa um lugar de destaque, chamando a atenção da comunidade científica, das autoridades médicas e governamentais de vários países, dentre eles, o Brasil. Dada as altas taxas de prevalência do câncer de colo uterino, a realização periódica do Papanicolau é considerada a melhor estratégia para a sua prevenção. O objetivo deste estudo é relatar a vivência de discentes do curso de enfermagem decorrida em uma unidade de Estratégia Saúde da Família da cidade de Belém, Pará, ocorrida através da efetuação de uma atividade de educação em saúde sobre o exame Papanicolau. Trata-se de um estudo descritivo com abordagem qualitativa, do tipo relato de experiência, onde a metodologia adotada perpassou pela Teoria da Problematização através da utilização do Arco de Maguerez. Evidenciou-se que algumas das mulheres participantes desconheciam o exame, outras já sabiam de sua existência, mas ainda não entendiam a importância de sua realização. Dentre as participantes, poucas já haviam realizado o exame, sendo que ainda apresentavam algumas dúvidas sobre ele. Acredita-se que que o objetivo da ação foi alcançado, uma vez que as mulheres que participaram destas atividades foram sensibilizadas quanto a relevância da realização do exame, bem como acerca da autonomia em saúde e da importância de um autocuidado diligente e eficaz para a manutenção da saúde e qualidade de vida.

Palavras-chave: Teste de Papanicolau; Neoplasias do colo do útero; Saúde da mulher; Enfermagem.

\begin{abstract}
Cervical cancer is a malignant neoplasm, which occupies a prominent place, drawing the attention of the scientific community, the medical and governmental authorities of several countries, particularly Brazil. Given the high prevalence rates of cervical cancer in the female population, periodic Pap smear is considered the best strategy for its prevention. The objective of this study is to report an experience of students of the nursing course that took place in a Family Health Strategy (FHS) unit in the city of Belém, which occurred through the performance of a health education activity on PAP. This is a descriptive study with a qualitative approach, of the experience report type, where the adopted methodology passed through the Theory of Problematization through the use of the Maguerez Arch. It became evident that some of the participating women were unaware of the examination, others already knew of its existence, but still did not understand the importance of its performance, among the participants, few had already performed the examination, but still had some doubts about it. It was bought from this article that the objective of the action was achieved, since the women who participated in these activities were sensitized as to the relevance of the exam, as well as about the autonomy in health and the importance of diligent and effective self-care. maintaining quality of life.
\end{abstract}

Keywords: Papanicolaou test; Uterine cervical neoplasms; Women's health; Nursing.

\section{Resumen}

El cáncer de cuello uterino es una neoplasia maligna, que ocupa un lugar destacado, llamando la atención de la comunidad científica, las autoridades médicas y gubernamentales de varios países, particularmente Brasil. Dadas las altas tasas de prevalencia de cáncer de cuello uterino en la población femenina, la prueba de Papanicolaou periódica se considera la mejor estrategia para su prevención. El objetivo de este estudio es reportar una experiencia de estudiantes del curso de enfermería que se llevó a cabo en una unidad de Estrategia de Salud de la Familia (ESF) en la ciudad de Belém, que ocurrió a través de la realización de una actividad de educación en salud en la UCPC. Se trata de un estudio descriptivo con enfoque cualitativo, del tipo relato de experiencia, donde la metodología adoptada pasó por la Teoría de la Problematización mediante el uso del Arco de Maguerez. Se hizo evidente que algunas de las mujeres participantes desconocían el examen, otras ya sabían de su existencia, pero aún no comprendían la importancia de su realización, entre las participantes, pocas ya habían realizado el examen, pero aún tenían algunas dudas sobre eso. De este artículo se compró que se logró el objetivo de la acción, ya que las mujeres que participaron en estas actividades fueron sensibilizadas sobre la relevancia del examen, así como sobre la autonomía en salud y la importancia de una autogestión diligente y eficaz. cuidado manteniendo la calidad de vida.

Palabras clave: Prueba de Papanicolaou; Neoplasias del cuello uterino; Salud de la mujer; Enfermería.

\section{Introdução}

O câncer cervical é uma neoplasia maligna que ocupa um lugar de destaque, chamando a atenção da comunidade científica, das autoridades médicas e governamentais de vários países, particularmente, do Brasil (Campos et al., 2017). A 
estimativa mundial aponta que o câncer do colo do útero foi o quarto mais frequente em todo o mundo, com uma estimativa de 570 mil casos novos, representando 3,2\% de todos os cânceres. Esse valor corresponde a um risco estimado de 15,1/100 mil mulheres (Saúde \& Silva, 2019).

Estimativas do Instituto Nacional do Câncer José Alencar Gomes da Silva - INCA, para os anos de 2020 a 2022, apontam o câncer de mama como o mais incidente e o câncer de colo de útero ocupando a quarta posição, desconsiderando a pele não melanoma. A estimativa anual no referido biênio para o câncer de mama é de 66.280 casos novos e um risco estimado de 81,06 casos por 100 mil mulheres. Para o câncer do colo do útero, a estimativa anual é de 16.590, com risco estimado de 15,43 casos em 100.000 mulheres (Ministério da Saúde, 2019).

Dada as altas taxas de prevalência do câncer de colo uterino, a realização periódica do Papanicolau é considerada a melhor estratégia para a prevenção, e deve ser disponibilizado às mulheres com vida sexual ativa, na faixa etária de 25 a 64 anos, que é considerada a de maior incidência desse tipo de câncer (Campos et al., 2017).

O rastreamento do câncer do colo do útero se baseia na história natural da doença e no reconhecimento de que o câncer invasivo evolui a partir de lesões precursoras (lesões intraepiteliais escamosas de alto grau e adenocarcinoma in situ), que podem ser detectadas e tratadas adequadamente, impedindo a progressão para o câncer (INCA, 2021).

O método principal e mais amplamente utilizado para rastreamento do câncer do colo do útero é o teste de Papanicolau (exame citopatológico do colo do útero). Segundo a Organização Mundial da Saúde (OMS), com uma cobertura da população-alvo de, no mínimo, $80 \%$ e a garantia de diagnóstico e tratamento adequados dos casos alterados, é possível reduzir, em média, de 60 a 90\% a incidência do câncer cervical invasivo (INCA, 2021).

As práticas da prevenção do câncer do colo do útero (PCCU), ainda hoje, representam um importante desafio de saúde pública. As razões para explicar este problema são as mais variadas, entre elas, os fatores culturais, sociais, econômicos e comportamentais, bem como a própria organização dos serviços públicos de saúde (Oliveira \& Pinto, 2007).

Outro aspecto importante diz respeito à dimensão psicossocial na realização do Papanicolau. Com efeito, são apontadas as dificuldades encontradas pelas mulheres na realização do exame, tais como: o medo dos resultados e de um possível diagnóstico de câncer; a falta de privacidade nos exames e de humanização no atendimento; a falta de sensibilização profissional para as rotinas de exames, e a baixa prioridade do profissional no atendimento integral às mulheres, revelando sentimentos como vergonha, medo e insegurança (Duavy et al., 2007).

Perante o exposto, o objetivo deste estudo é relatar a vivência de discentes do curso de enfermagem decorrida em uma unidade de Estratégia Saúde da Família (ESF) da cidade de Belém/PA, ocorrida através da efetuação de uma atividade de educação em saúde, com a finalidade de esclarecer possíveis dúvidas e anseios das mulheres acerca da realização do exame Papanicolau.

\section{Metodologia}

Trata-se de um estudo descritivo com abordagem qualitativa, do tipo relato de experiência, que ocorreu a partir de vivências de acadêmicos de enfermagem em uma ação de educação em saúde em uma Estratégia Saúde da Família, no município de Belém-Pará.

A metodologia adotada perpassou pela Teoria da Problematização através da utilização do Arco de Maguerez, composta de cinco etapas complementares que visam o estudo de uma determinada realidade para a posterior intervenção voltada a mitigação das problemáticas encontradas, dividindo-se em: observação da realidade, levantamento de pontos-chave, teorização, desenvolvimento das hipóteses de solução e, por fim, retorno com aplicação à realidade (Berbel, 2011).

Inicialmente, observou-se durante as práticas do componente curricular "Enfermagem em Saúde da Mulher" que a unidade contava com baixa adesão ao exame preventivo do câncer de colo uterino. Além disso, notou-se durante as consultas 
grande desinformação das mulheres usuárias do local quando indagadas sobre a realização do exame, bem como a pouca compreensão sobre a sua importância.

Dessa maneira, juntamente com os docentes preceptores da prática, optou-se por trabalhar esse assunto dentro da atenção básica, uma vez que este local é propício para o desenvolvimento de ações educativas. Para tanto, buscou-se na literatura a base para o delineamento da atividade, com leituras de artigos referentes à temática, visando fornecer maior rigor científico para as informações que seriam repassadas, bem como auxiliar na escolha das etapas a serem efetivadas no local.

Para tanto, optou-se por trabalhar com tecnologias leves, por meio da utilização de cartazes e a demonstração dos instrumentos utilizados para a realização do exame, bem como a construção de uma dinâmica para o fomento ao autocuidado e a manutenção da qualidade de vida, a fim de incentivar a promoção da saúde das mulheres participantes.

No retorno à realidade, a ação ocorreu através das seguintes etapas: convite para participação da ação para as usuárias que aguardavam consulta no local, acolhimento inicial com explicação da importância da participação das mulheres e objetivo das atividades, roda de conversa com as orientações gerais sobre a doença, formas de transmissão, prevenção e sintomatologia, além da demonstração dos instrumentos utilizados no exame. Ao final houve uma dinâmica para o fomento ao autocuidado e promoção da interação social entre as usuárias do serviço.

\section{Resultados}

No dia da ação, inicialmente o grupo de discentes percorreu a unidade em busca das participantes, sendo que 17 mulheres aceitaram participar após receberem informações sobre o objetivo da atividade. Notou-se, em um primeiro momento, que a grande maioria delas se encontravam retraídas e tímidas em conversar sobre o assunto, porém, à medida que a ação decorreu, elas se tornaram mais participativas e interessadas.

Durante o acolhimento inicial, o grupo se apresentou e explicou de maneira geral a importância da participação das mulheres na atividade. Posteriormente, durante a roda de conversa, foram abordados os principais aspectos da patologia, suas formas de transmissão, sintomatologia e os exames preventivos, com foco no PCCU.

Neste ínterim, evidenciou-se que algumas mulheres desconheciam o exame, outras já sabiam de sua existência, mas ainda não entendiam a importância de sua realização. Dentre as participantes, apenas quatro já haviam realizado o exame, sendo que ainda apresentavam algumas dúvidas sobre ele. Ao passo que os questionamentos iam surgindo, os acadêmicos, juntamente com os docentes que os acompanhavam, esclareciam e reiteravam a importância do exame na saúde da mulher.

Além disso, durante os relatos obtidos na roda de conversa, uma das participantes referiu ter tido um caso de câncer de colo uterino na família, enfatizando os sentimentos vivenciados por essas mulheres, como a vergonha, o desconhecimento e o medo que envolve um diagnóstico tardio e um prognóstico desfavorável. Neste momento, ficou evidente a comoção por parte das outras participantes, que confortaram aquela que estava relatando o caso.

Ademais, após esses esclarecimentos, houve uma dinâmica para incentivar o autocuidado e a manutenção da qualidade de vida dessas mulheres, na qual cada uma escreveria em uma caixinha confeccionada pelos acadêmicos frases de incentivo e cuidados para a participante que se encontrava ao lado, que iria ler em voz alta e apresentar suas considerações sobre a fala.

Durante a realização desta etapa da ação, notou-se que as usuárias se encontravam empolgadas e participativas na construção das frases, auxiliando umas às outras no desenvolvimento da atividade. No momento da leitura, percebeu-se que a maioria das frases eram de incentivo a manutenção de hábitos saudáveis, saúde mental e qualidade de vida, além de falas com viés espirituais e resilientes. As mulheres mostraram-se sensibilizadas durante a leitura, auxiliando no desenvolvimento do autocuidado e do empoderamento deste público.

Por fim, as participantes agradeceram pela ação realizada e a troca de conhecimentos. Muitas das participantes 
afirmaram que iriam marcar seu primeiro exame em breve na unidade, além de se comprometer em disseminar as informações compartilhadas na atividade para seus familiares e amigos, reiterando a relevância desta ação educativa na disseminação dos conhecimentos e práticas em saúde.

\section{Discussão}

Evidencia-se a partir deste relato que a ausência de conscientização acerca de um problema pode se tornar um obstáculo para o sucesso de ações, que objetivem contemplar grandes grupos. Dessa forma, as ações educativas podem ser melhor utilizadas tanto em grupos de mulheres, como na atenção individualizada, na busca de uma melhor compreensão das informações divulgadas e na conscientização da importância da prevenção e detecção precoce. Uma vez que, seja durante a consulta de enfermagem ou em oportunidades de realização de atividades de educação em saúde, é fundamental que se esclareçam as dúvidas existentes acerca do exame Papanicolau, objetivando intensificar o conhecimento por parte das mulheres e minimizar medos e constrangimentos que possam existir na realização do exame (Souza, 2011).

Posto isso, verifica-se que a educação em saúde é forte aliada no objetivo de despertar mudanças de comportamento. Por intermédio dela, as usuárias do sistema de saúde podem adquirir informação, refletir sobre suas práticas, bem como sobre a importância do exame ginecológico e da autovalorização. Ela é uma prática social que contribui para a formação da consciência crítica das pessoas a respeito de seus problemas de saúde, levando em conta a sua realidade (Rodrigues et al., 2012).

A promoção da saúde visa assegurar a igualdade de oportunidades e proporcionar os meios que permitam a todas às pessoas realizar completamente seu potencial de saúde. Os indivíduos e as comunidades devem ter oportunidade de conhecer e controlar os fatores determinantes da sua saúde. Ambientes favoráveis, acesso à informação, habilidades para viver melhor, bem como oportunidades para fazer escolhas mais saudáveis, estão entre os principais elementos capacitantes. Os profissionais e os grupos sociais, assim como as equipes de saúde, têm a responsabilidade de contribuir para a mediação entre os diferentes interesses, em relação à saúde, existentes na sociedade (Czeresnia \& Freitas, 2020).

Trabalhar com educação popular é a maneira mais precisa de se conhecer uma realidade e poder através de observações e atividades lúdicas perceber as necessidades existentes e a partir desse conhecimento obtido, buscar dispositivos para uma transformação social sem interferir diretamente no cotidiano dos indivíduos (Souza et al., 2015).

Na detecção precoce do câncer esta é a arma e a estratégia fundamental para o seu controle. Para que isso ocorra, é necessário que a mulher voluntariamente procure os serviços de saúde para se submeter ao exame preventivo. Esse comportamento voltado para a saúde é um ato aprendido que necessita de uma ação educativa, de um convencimento. Assim, uma mudança nesse perfil epidemiológico depende não só das mulheres e nem só das políticas governamentais, ou só das ações dos profissionais de saúde, mas sim de todos eles, numa estratégia articulada volta da para a educação em saúde e para a redução dos agravos à saúde da mulher (Pelloso et al., 2004).

Ademais, a enfermagem trabalha diretamente no exame do Papanicolau, sendo de grande importância que haja o compartilhamento das informações referentes a ele, bem como para os demais procedimentos e atividades desenvolvidas por estes profissionais. Tal atitude permite incentivar as estratégias de promoção da saúde e prevenção de agravos, ampliando o aprendizado da população (Lima et al., 2017).

Além disso, enfatiza-se a relevância da participação de acadêmicos nessas atividades, visto que a partir disso eles podem adquirir aptidões e desenvolver habilidades e competências que os auxiliarão em sua gestão de cuidados futuramente. Para mais, essa participação também influencia diretamente na construção de um olhar holístico e equânime do profissional, a partir do conhecimento de diferentes demandas e da aplicação de intervenções diretamente na comunidade, aumentando o contato com a realidade dos usuários dos serviços (Silva et al., 2020). 
Para mais, salienta-se que muitas das problemáticas enfrentadas atualmente pelas mulheres diante da realização do exame Papanicolau poderiam ser minimizadas se houvesse um comprometimento maior, por parte dos profissionais de saúde, no desenvolvimento de atividades educativas. Por vezes, estes profissionais acabam se limitando ao atendimento pontual da demanda existente, marcado pela consulta de enfermagem e realização de procedimentos e, consequentemente, essas mulheres ficam com seus questionamentos internalizados, deixando de realizar o exame por conta de seus medos e dúvidas que facilmente poderiam ser sanados.

\section{Considerações Finais}

A partir do exposto, ratifica-se a importância de ações de educação voltadas à promoção da saúde da mulher, em especial no âmbito da Atenção Básica de Saúde. Evidenciou-se, a partir deste artigo, que o objetivo da ação foi alcançado, uma vez que as mulheres que participaram destas atividades foram sensibilizadas quanto a relevância da realização do exame, bem como acerca da autonomia em saúde e da importância de um autocuidado diligente e eficaz para a manutenção da saúde e qualidade de vida.

Ademais, reitera-se a relevância da participação de acadêmicos de enfermagem em ações fora do contexto universitário, com foco na resolução de problemas reais e atuação direta dentro da comunidade, cuja realização promove a obtenção paulatina de habilidades e competências necessárias a atuação deste futuro profissional, possibilitando a criação de um olhar holístico e humanizado.

Por fim, espera-se que este trabalho possa embasar estudos futuros, possibilitando o desenvolvimento de estratégias que auxiliem na prevenção do câncer de colo uterino, bem como na promoção da qualidade de vida da mulher brasileira. Ademais, como sugestão para trabalhos futuros, salienta-se a necessidade de se trabalhar essa temática em todos os níveis assistenciais, em unidades de saúde variadas e com maior quantidade de participantes, possibilitando, assim, a disseminação em longa escala das informações acerca da prevenção desta patologia, bem como maior variabilidade de resultados.

\section{Referências}

Berbel, N. A. N. (2011). As metodologias ativas e a promoção da autonomia de estudantes. Semina: Ciências Sociais e Humanas, 32(1), 25-40.

Campos, E. A. D., Castro, L. M. D., \& Cavalieri, F. E. D. S. (2017). "Uma doença da mulher”: experiência e significado do câncer cervical para mulheres que realizaram o Papanicolau. Interface - Comunicação, Saúde, Educação, 21(61), 385-96.

Czeresnia, D., \& Freitas, C. M. d. (2020). Promoção da Saúde: conceitos, reflexões, tendências (6th ed.). Fiocruz.

Duavy, L. M., Batista, F. L. R., Jorge, M. S. B., \& Santos, J. B. F. (2007). A percepção da mulher sobre o exame preventivo do câncer cérvico-uterino: estudo de caso. Ciência e Saúde Coletiva, 2(3),733-42.

INCA. Detecção Precoce. (2021). Instituto Nacional do Câncer. https://www.inca.gov.br/en/node/1194.

Lima, H. D. F., Lima, S. M. D., Ribeiro, G. C., \& Lima, L. R. D. (2017). Saúde e educação popular com mulheres sobre o exame papanicolau: relato de experiência. EEDIC, 4(1), 1-5.

Ministério da Saúde (BR). Instituto Nacional de Câncer José Alencar Gomes da Silva. Estimativa 2020: câncer de câncer no Brasil. Rio de Janeiro: INCA. 120 p. https://www.inca.gov.br/sites/ufu.sti.inca.local/files/media/document/estimativa-2020-incidencia-de-cancer-no-brasil.pdf

Oliveira, M. M. D., \& Pinto, I. C. (2007). Percepção das usuárias sobre as ações de Prevenção do Câncer do Colo do Útero na Estratégia Saúde da Família em uma Distrital de Saúde do município de Ribeirão Preto, São Paulo, Brasil. Revista Brasileira de Saúde Materno Infantil, 7(1), 31-38.

Pelloso, S. M., Carvalho, M. D. B., Higarashi, I. H. (2004). Conhecimento das mulheres sobre o câncer cérvico-uterino. Acta Scientiarum Health Sciences, 26(2), 319-24.

Rodrigues, B. C., Carneiro, A. C. M. D. O., Silva, T. L. D., Solá, A. C. N., Manzi, N. D. M., Schechtman, N. P., Magalhães, H. L. G. D. O., \& Dytz, J. L. G. (2012). Educação em Saúde para a Prevenção do Câncer Cérvico-uterino. Revista brasileira de educação médica, 36(1), $149-154$.

Saúde, M. D., \& Silva, I. N. D. C. J. A. G. D. (2019). Estimativa 2020: incidência de câncer no Brasil. Biblioteca Virtual em Saúde Prevenção e Controle de Câncer. 
Research, Society and Development, v. 10, n. 3, e33310312818, 2021

(CC BY 4.0) | ISSN 2525-3409 | DOI: http://dx.doi.org/10.33448/rsd-v10i3.12818

Silva, J. M. L., Monteiro, A. J. C., Coutinho, E. S., Cruz, L. B. S., Araújo, L. T., Dias, W. B, \& Costa, P. V. D. P. (2020). O brinquedo terapêutico instrucional como ferramenta na assistência oncológica infantil. Research, Society and Development, 9 (7): 1-14.

Souza, G. G. (2011). A importância de ações educativas para prevenção do câncer de colo de uterino no contexto da estratégia saúde da família [Trabalho de Conclusão de Curso, Universidade Federal de Minas Gerais].

Souza, K. R. D., Paixão, G. P. D. N., Almeida, E. D. S. D., Sousa, A. R. D., Lirio, J. G. D. S., \& Campos, L. M. (2015). Educação popular como instrumento participativo para a prevenção do câncer ginecológico: percepção de mulheres. Revista Cuidarte, 6(1), 892-9. 